\title{
Associations between red and processed meat consumption and cardio-metabolic risk markers among British adults
}

\author{
Ditte A. Hobbs $\mid$ Julie A. Lovegrove $\mid$ D. Ian Givens
}

\section{Introduction}

SACN recommends the consumption of $\leq 70 \mathrm{~g}$ /day of red and processed meat ${ }^{[1]}$. Red and processed meat ( $\geq 100 \mathrm{~g} /$ day $)$ has been associated with an increased risk of colon cancer ${ }^{[2]}$. The aim of this study was to examine the association between diets with varying levels of red meat (RM), processed red meat (PRM) and total red and processed meat (TRPM) with cardiovascular health markers in adults from the UK National Diet and Nutrition Survey (NDNS).

\section{Methods}

\section{Sample and study design}

- This study was a secondary analysis of data from the nationally representative NDNS (2008/09-2011/12) $)^{[3]}$.

- This cross-sectional survey included data from 6,828 persons aged $\geq 1.5$ y (of whom 2,697 were adults aged 19-64 y).

Dietary assessment

- Dietary intakes were obtained from 4-day diet diaries.

- Participants were classified into tertiles according to their RM, PRM and TRPM consumption, with tertile 1 (T1) and tertile 3 (T3) representing diets with the lowest and highest intakes, respectively.

- $\quad \mathrm{RM}$ - beef, lamb, pork, burgers and other red meat.

- PRM - processed red meat and sausages (includes cured and canned meats, and meats containing preservatives).

Markers of cardiovascular health

- Anthropometric measures, blood pressure, pulse pressure, plasma glucose, glycated haemoglobin (HbAlc), homocysteine, C-reactive protein (CRP), triacylglycerol (TAG), total cholesterol (T-chol), high-density cholesterol (HDL-C) and low-density cholesterol (LDL-C).

Analytical sample

- Participants with diabetes $(n=9)$, fasted blood glucose above 7 $\mathrm{mmol} / \mathrm{L}(n=5)$, taking medicines known to affect blood analytes $(n=925)$ or missing data $(n=690)$ were excluded. Therefore, a total number of 1068 participants ( $\mathrm{M} n=481, \mathrm{~F} n=587$ ) were included in this analysis, unless otherwise stated.

\section{Statistical analysis}

- ANCOVA was used to detect statistically significant differences between tertiles of RM, PRM and TRPM consumption and cardio-metabolic risk markers controlling for age, sex, energy intake (kJ), BMI, social class and smoking status (model 1) and dietary vitamin $\mathrm{C}$ (biomarker of fruit and vegetable intake), SFA and total fat intakes (model 2).

- Bonferroni post-hoc tests were used to detect differences between tertiles.

- A $P$-value of $\leq 0.05$ was considered statistically significant.

\section{Results and Discussion}

\section{Associations with cardiovascular health markers}

Total red and processed meat (TRPM) consumption

- There was a significant difference across TRPM tertiles for:

- Glucose (model 1: $P=0.004$; model 2: $P=0.001$ )

- CRP (model 1: $P=0.031$ )

- Larger waist circumference (model 1: $P=0.002$; model 2: $P=0.007$ )

- Larger waist to hip ratio (model 1: $P=0.005$; model 2: $P=0.016$ )
- The diets of participants in the highest tertile of TRPM (T3; mean $131.3 \mathrm{~g}$ /day (SD 43.2)) compared with (T1; mean $18.4 \mathrm{~g} /$ day (SD 14.9)) were associated with significantly higher concentrations of (model 2):

- Glucose $(P$-T2/T1 $=0.012, P$-T2/T3=0.004 $)$.

- Waist circumference $(P-\mathrm{T} 1 / \mathrm{T} 3=0.075)$.

- Waist to hip ratio $(P-\mathrm{T} 2 / \mathrm{T} 3=0.012)$.

Processed red meat (PRM) consumption

- There was a significant difference across tertiles of PRM consumption for:

- Homocysteine (model 1: $P=0.027$; model 2: $=0.039$ )

- Glucose (model 1: $P=0.013$; model 2: $P=0.018$ )

- HbA1c (model 1: $P=0.0001$; model 2: $P=0.0001$ )

- The diets of participants in the highest tertile of PRM (T3; mean $75.9 \mathrm{~g} /$ day (SD 32.0)) compared with (T1; mean $2.7 \mathrm{~g} /$ day (SD 4.2)) were associated with significantly higher concentrations of (model 2):

- Homocysteine $(P-\mathrm{T} 1 / \mathrm{T} 3=0.044)$

- Glucose $(P-\mathrm{T} 1 / \mathrm{T} 3=0.018)$

- $\operatorname{HbA} 1 \mathrm{c}(P-\mathrm{T} 2 / \mathrm{T} 1=0.001$ and $P$-T2/T3=0.001 $)$

TABLE 1 Adjusted biomarkers of cardiovascular health according to tertile PRM intake ${ }^{1}$

\begin{tabular}{|c|c|c|c|c|c|c|}
\hline \multirow[b]{2}{*}{ Biomarkers } & \multirow[b]{2}{*}{$n$} & \multicolumn{3}{|c|}{ Tertiles of PRM } & \multicolumn{2}{|c|}{$P$-value ${ }^{2}$} \\
\hline & & 1 & 2 & 3 & Model 1 & 1 Mode \\
\hline T-cholesterol $(\mathrm{mmol} / \mathrm{L})$ & 695 & $5.1(5.0,5.3)$ & $5.1(5.0$ & $5.2(5.1,5.3)$ & 0.47 & 0.577 \\
\hline HDL-C (mmol/L) & 695 & $1.5(1.4$ & 6 & 5) & 0 . & 12 \\
\hline DL-C (mmo & 689 & $3.2(3.1$, & $1 / 30$ & 3) & 0. & 0.65 \\
\hline $\mathrm{AG}(\mathrm{mmol} / \mathrm{L})$ & 695 & $1.2(1.1$, & $3(1.1$, & $4(1.3$ & 0.2 & 0.11 \\
\hline $\mathrm{RP}(\mathrm{mg} / \mathrm{L})$ & 696 & $2.6(2.0$ & $7(2.2$ & $2.7(2.1$ & 0.99 & 0.96 \\
\hline bA1c (\%) & 685 & $5.4(5.4$, & $5.3(5.3$ & $5.4(5$. & 0.0001 & 0.0001 \\
\hline lucose $(n$ & 689 & $5.0(4.9$, & $5.0(4.9$, & $5.1(5.0$ & 0.0 & 0.018 \\
\hline omocysteine $(\mu \mathrm{mol} / \mathrm{L})$ & 677 & $9.4(8.7,10)$ & $10(9.5,11)$ & $1(9.9,11)$ & 0.027 & 0.039 \\
\hline \multicolumn{7}{|c|}{$\begin{array}{l}\text { IValues are weighted means }(95 \% \text { CIs) adjusted for age, gender, BMI, smoking status, social class, energy, } \\
\text { vitamin C, saturated fat and total fat intakes. CRP, c-reactive protein; Hb, haemoglobin; HbAls, glycated } \\
\text { haemoglobin; HDL-C, high-density lipoprotein cholesterol; LDL-C, low-density lipoprotein cholesterol, } \\
\text { TAG; triacylglycerol. 2Differences between health biomarkers across PRM tertiles using general linear } \\
\text { models. Model } 1 \text { was adjusted for age, sex, energy intake, social class, BMI and smoking and model } 2 \\
\text { additional adjustment was made foe dietary vitamin C, saturated fat and total fat intakes. } P=<0.05 \text { was } \\
\text { considered significant. }\end{array}$} \\
\hline
\end{tabular}

Red meat (RM) consumption

- Diets of participants in the highest tertile of RM (T3; mean 77.8 g/day (SD 32.4)) were associated with significantly lower pulse pressure compared with (T1; mean $1.7 \mathrm{~g} /$ day (SD 3.4)) (Model 1: $P$ trend $=0.022, \quad P$-T1/T3 $=0.017 ; \quad$ model $2: \quad P$-trend $=0.017, \quad P$ $\mathrm{T} 1 / \mathrm{T} 3=0.013)$.

\section{Conclusion}

The consumption of TRPM (mean $70.3 \mathrm{~g}$ /day (SD 54.0)), was associated with an increased number of cardiovascular disease risk markers, which was mainly driven by PRM intake. RM intake was only associated with lower pulse pressure.

References

1. Scientific Advisory Committee on Nutrition (SACN) (2011) Iron and Health Report.

https://www.gov.uk/government/publications/sacn-iron-and-health-report (accessed August 2017).

Vieira AR, Abar L, Chan DSM et al. (2017) Ann Oncol 8, 1788-1802.

Bates B, Lennox A, Prentice A et al. (2017) National Diet and Nutrition Survey (NDNS) - results from years $1-4$ (combined) of the rolling programme (2008/2009-2011/2012).

https://www.gov.uk/government/uploads/system/uploads/attachment data/file/594361/NDNS Y1 to 4 UK eport full text revised_February 2017.pdf (accessed August 2017).

Acknowledgements

This work was supported by the Agriculture and Horticulture Development Board (AHDB)

Contact information

Department of Food \& Nutritional Sciences, University of Reading, Whiteknights, RG6 6AH Email: d.a.hobbs@reading.ac.uk |www.reading.ac.uk/nutrition 